Salma Regina Rodrigues Balista ${ }^{1}$ Sílvia Maria Santiago ${ }^{2}$ Heleno Rodrigues Corrêa Filho²

\section{A atenção à saúde do trabalhador nas unidades bá- sicas de saúde do SUS: estudo de caso em Campinas, São Paulo}

\author{
Workers' Health service delivery by primary care units in the \\ Brazilian Unified Health System: a case-study in Campinas, \\ São Paulo, Brazil
}

1 Doutoranda do Departamento de Medicina Preventiva e Social da Faculdade de Ciências Médicas da Universidade Estadual de Campinas (Unicamp), Campinas, SP, Brasil.

2 Docente do Departamento de Medicina Preventiva e Social da Faculdade de Ciências Médicas da Universidade Estadual de Campinas (Unicamp), Campinas, SP, Brasil.

"Este artigo baseou-se na dissertação de mestrado de Salma Regina Rodrigues Balista intitulada $A$ descentralização da vigilância da saúde do trabalhador no SUS-Campinas, SP, apresentada ao Departamento de Medicina Preventiva e Social da Faculdade de Ciências Médicas da Unicamp, em 2008.

\section{Contato:}

Salma Regina Rodrigues Balista

Universidade Estadual de Campinas

Faculdade de Ciências Médicas,

Departamento de Medicina Preventiva e Social

Rua Tessália Vieira de Camargo, 126, Barão Geraldo, Campinas, SP, Brasil CEP 13083-970

E-mail:

salmarrb@fcm.unicamp.br

\section{Resumo}

Objetivo: avaliar o processo de implantação das ações de vigilância em saúde do trabalhador nas Unidades Básicas de Saúde (UBS) do SUS, em Campinas, SP. Métodos: foram usados métodos qualitativos e quantitativos para produzir uma aproximação sob diferentes aspectos do objeto em estudo. Os acidentes e as doenças relacionados ao trabalho registrados em 2006 foram eleitos para verificar se as UBS conduziram ações de vigilância e se ocorreram mudanças preventivas e protetoras no processo de trabalho e no ambiente. Realizamos entrevistas com os profissionais responsáveis pela vigilância em saúde do trabalhador das unidades. Resultados: as ações de atenção à saúde e a notificação foram implantadas com êxito principalmente para acidentes de trabalho. Todavia, o vínculo das doenças relacionadas com o trabalho foi menos reconhecido. Os acidentes e as doenças receberam uma abordagem burocrática, negligenciando a relevância epidemiológica para a tomada de decisão. Trabalhadores do mercado informal não receberam ações específicas. A gestão dos sistemas locais de saúde ainda necessita de regras específicas para estabelecer seu papel na integração dos sistemas de informação com a vigilância da saúde do trabalhador na atenção primária. Conclusão: o presente estudo apontou que a descentralização da vigilância da saúde do trabalhador para a atenção primária foi parcialmente efetivada em Campinas.

Palavras-chave: saúde do trabalhador; vigilância em saúde do trabalhador; avaliação em saúde; avaliação de programas e projetos de saúde; vigilância.

\begin{abstract}
Objective: The purpose of this study was to analyze the implementation of workers' health and epidemiological surveillance services at the primary healthcare units (UBS) of the Brazilian Unified Health System (SUS) in Campinas, São Paulo, Brazil. Method: Qualitative and quantitative methods were combined to yield a parallax approach to the research object. Work-related injuries and diseases recorded in 2006 were selected to investigate whether UBS conducted surveillance actions and if preventive and protective changes had occurred in work processes and environment. Professionals in charge of workers' health surveillance in the units were interviewed. Results: The study showed that healthcare and disease notification were successfully implemented, mainly those with the purpose of reporting work injuries. Nevertheless, poor recognition was given to the causal links of workrelated diseases; they were dealt bureaucratically, and their epidemiologic relevance for decision taking was neglected. Informal workers did not receive specific attention. Administration of local health units still demands specific protocols to establish their role in integrating information systems to workers' health surveillance in primary care level. Conclusion: Decentralization of workers' health service was only partially implemented in the primary healthcare units in Campinas.
\end{abstract}

Keywords: occupational health; worker's health surveillance; health assessment; surveillance; program evaluation. 


\section{Introdução}

Ao estudarmos o que alguns autores apresentam acerca da descentralização e da integralidade na atenção à saúde, podemos verificar uma relação entre este princípio e aquela diretriz do Sistema Único de Saúde (SUS), reconhecendo a descentralização como uma das formas de buscar a integralidade do sistema de saúde na medida em que proporciona a aproximação dos serviços e dos profissionais de saúde das pessoas e da realidade local (MERHY, 1997; CECÍLIO, 2001; MATTOS, 2001; CAMPOS, 2003; SILVA-JÚNIOR; MERHY; CARVALHO, 2003).

Podemos entender a integralidade no espaço da unidade básica de saúde (UBS) ${ }^{3}$ como o esforço das equipes de serviços em compreender e buscar respostas para as necessidades de saúde que o usuário apresenta, muitas vezes complexas. A essa integralidade, Cecílio (2001) dá o nome de "integralidade focalizada". A expressão "integralidade ampliada", utilizada por esse autor, traz a ideia de que a pessoa com necessidades de saúde - foco principal e razão de ser dos serviços de saúde - não conseguirá ter todas elas atendidas por um único serviço de saúde, podendo vê-las respondidas e atendidas somente de maneira articulada com uma rede de serviços dentro e fora do setor saúde. As diferentes tecnologias em saúde estão disponibilizadas em uma ampla rede de serviços e, para atender a algumas necessidades, é preciso uma ação intersetorial (CECÍLIO, 2001).

Neste sentido, parece-nos que a diretriz do SUS para a descentralização pode favorecer esse resultado.

\section{Ações de Vigilância em Saúde do Trabalhador}

A organização do sistema local de saúde em unidades com responsabilidade sobre uma área de abrangência e com atribuições de realizar ações de Vigilância em Saúde (Visa) facilita a identificação de problemas locais de saúde a serem enfrentados com os recursos existentes na rede de saúde e em outros setores.

As práticas ampliadas de Visa permitem a identificação de problemas de saúde que afetam grupos populacionais de determinados territórios. O conhecimento da realidade de um território, seus aspectos demográficos, socioeconômicos, políticos, culturais, epidemiológicos e sanitários, contribui para a compreensão da relação entre as condições de vida e o processo saúde-doença, bem como para o acesso a ações e serviços de saúde e, ainda, favorece a tomada de decisão para as intervenções necessárias (TEIXEIRA; PAIM; VILASBOAS, 2002).

A Vigilância em Saúde do Trabalhador (Visat) é uma prática social que, ao ser incorporada à Visa, requer "uma compreensão transdisciplinar e uma ação transversal inter e intra-setorial", ou seja, que deve ser exercida a muitas mãos, pelos diferentes atores que tenham envolvimento com a questão, extrapolando o setor saúde. Essa concepção decorre da influência política, técnica e científica da medicina social latino-americana e da experiência italiana de reforma sanitária. A Visat congrega diferentes componentes estruturais para a abordagem interdisciplinar da relação processo de trabalho e saúde: componentes tecnológicos utilizados em determinado processo de produção; epidemiológicos para a avaliação de risco; e sociais, como as condições econômicas e de organização dos trabalhadores, incorporando o processo de trabalho como categoria e objeto fundamentais para sua análise e intervenção. Busca o conhecimento para a ação e, com isso, também gera novos conhecimentos (MACHADO, 1997; MACHADO, 2005).

A abordagem territorial é facilitadora da ação de Visat, pois estimula e permite o conhecimento dos riscos presentes em determinada área geográfica, como a área de abrangência de uma unidade de saúde ou macrorregiões e também a atuação diferenciada para cada um deles, possibilitada por uma aproximação dos determinantes desses agravos e riscos (MACHADO, 1997).

A equipe de saúde, que conhece seus indicadores e os utiliza para planejar melhor suas ações de intervenção, pode identificar e priorizar grupos mais vulneráveis, por exemplo, trabalhadores expostos a riscos.

Contudo, não é possível realizar essas medidas em posição distante da realidade local. Seu equacionamento dar-se-á mais adequadamente estando-se muito próximo do problema. Novamente, é implícita a descentralização como facilitadora das mudanças de modelo de atenção capazes de responder mais adequadamente às necessidades de saúde da população.

\section{A Vigilância em Saúde do Trabalhador em Campinas}

O SUS de Campinas, SP, considera a UBS como pilar estruturante e conta com um sistema de vigilância atuante desde antes mesmo da municipalização. Nessa lógica, o município assumiu responsabilidades sobre ações de vigilância e de saúde do traba-

\footnotetext{
${ }^{3}$ No SUS brasileiro, as redes assistenciais locais hierarquizadas são constituídas por Centros de Saúde (CS) primários e secundários. Os CS primários são porta de entrada do Sistema e também são chamados de Unidades Básicas de Saúde (UBS). Os CS secundários são chamados de Centros de Referência e recebem usuários enviados pelas UBS para diagnóstico e tratamento especializado. Neste texto, CS primário e UBS são sinônimos. A palavra "básica" não significa que não sejam realizados procedimentos complexos nas UBS.
} 
lhador e promoveu, na sequência, a descentralização de algumas dessas ações para as equipes distritais e locais de saúde.

As ações de vigilância epidemiológica foram assumidas integralmente pelo município de Campinas desde 1987 com a criação do Grupo Municipal de Vigilância Epidemiológica, antes da municipalização formal, que ocorreu em 1988 (SAGLIONI; BALISTA, 2004).

O Programa de Saúde do Trabalhador (PST) foi criado no município de Campinas em 1987 por iniciativa de sindicatos, da Universidade Estadual de Campinas e dos governos municipal e estadual e deveria exercer suas atividades em três áreas: assistência à saúde, vigilância e educação - sendo a Visat entendida como instrumento fundamental para a identificação e a intervenção em situações de risco à saúde relacionadas ao trabalho (MEDEIROS, 2001).

A partir de 1989, iniciou-se um processo intenso de capacitação e descentralização da vigilância epidemiológica para as UBS, com a incorporação da função do "vigilante local" ou da "moça da vigilância", que, juntamente com a coordenação da unidade, respondia pela organização e a execução dessas ações em nível local (ABRAHÃO, 1994; SAGLIONI; BALISTA, 2004; VILELA, 2005).

Para dar amparo jurídico às ações, foi promulgada a Lei Municipal no 6.764/91, que autorizou o Executivo Municipal a observar as legislações federal e estadual concernentes às ações de vigilância sanitária, vigilância epidemiológica, de fiscalização da alimentação pública e da nutrição, de fiscalização do saneamento, do meio ambiente e da saúde do trabalhador. A sua regulamentação estabeleceu os processuais administrativos e definiu a figura da autoridade sanitária constituída nos diversos níveis da Secretaria de Saúde, ou seja, das UBS ao nível central (CAMPINAS, 1991; ABRAHÃO, 1994; SAGLIONI; BALISTA, 2004).

A vigilância sanitária já era executada pelo município em relação ao comércio varejista de alimentos e ao saneamento, mas foi oficialmente municipalizada no final de 1992 para a quase totalidade dos objetos de atuação, com exceção dos serviços de hemodiálise, dos bancos de sangue, órgãos e tecidos. Imediatamente após, no ano de 1993, quando a Secretaria assume irreversivelmente a estruturação dos distritos sanitários, foram formadas cinco equipes distritais de Visa e, assim, foram descentralizadas e unificadas as ações de vigilância sanitária e epidemiológica para essas equipes, constituindo-se autoridades sanitárias distritais (ABRAHÃO, 1994; SAGLIONI; BALISTA, 2004).
Também em 1993, o PST passou a atuar como Centro de Referência de Saúde do Trabalhador CRST (MEDEIROS, 2001).

Em 1994, iniciou-se a descentralização para a rede básica do atendimento de acidentes de trabalho que não necessitassem de atendimento hospitalar. Esse atendimento compreendia a prestação de cuidados de assistência médica e de enfermagem e o respectivo preenchimento e encaminhamento da Comunicação de Acidente de Trabalho (CAT). Essa medida fazia parte da readequação do papel do CRST à época e da rede básica (MEDEIROS, 2001).

Houve capacitação da rede básica para assumir o atendimento dos acidentes de trabalho, que incluiu as orientações sobre preenchimento e fluxo da CAT entre os serviços da Secretaria Municipal de Saúde e o Instituto Nacional de Seguridade Social - INSS (CAMPINAS, 1997, CAMPINAS, 2006a).

O intuito era dar condições ao CRST de realizar sua missão de prestar atenção à saúde do trabalhador, desafogando o atendimento médico. $\mathrm{O}$ outro ganho com a descentralização foi diminuir a via crucis dos trabalhadores, que tinham de peregrinar entre serviços de saúde, empresa e INSS (MEDEIROS, 2001, p. 128). Contudo, segundo Medeiros (2001), a descentralização ocasionou piora na qualidade dos registros das informações no município, com comprometimento das ações de intervenção coletivas. Em 1998, inicia-se a descentralização da assistência às doenças do trabalho como a única maneira de desafogar o CRST da avalanche de atendimentos a casos de lesões por esforços repetitivos (LER) e realizar ações preventivas, tendo como pano de fundo dessa discussão a rede básica como porta de entrada do sistema de saúde. Com capacitação da rede, retaguarda laboratorial e retaguarda técnica do CRST, isso seria possível. A descentralização da assistência não faria sentido se fosse descolada da descentralização da intervenção nos ambientes de trabalho (MEDEIROS, 2001). Esse processo iria se efetivar em 2006, com a implantação do Plano de Ação e Metas da Saúde do Trabalhador no município e, seguindo as orientações da Rede Nacional de Saúde do Trabalhador (Renast), nesse mesmo ano, passa a adotar a denominação de Cerest, indicada pela Renast para todos os Centros de Referência de Saúde do Trabalhador no Brasil (BRASIL, 2005; CAMPINAS, 2006b).

Assim, no município de Campinas, as ações de Visa devem ser desenvolvidas em alguma medida em todas as unidades básicas que contam com o apoio técnico das equipes distritais de Visa do Cerest e da Coordenadoria de Vigilância em Saúde (Covisa) para a complementação ou execução de ações mais complexas. 
O objetivo do presente estudo foi avaliar o processo de implantação da diretriz da descentralização da Visat para as UBS do município de Campinas, incorporando-as às ações locais da Visa.

\section{Método}

Esta pesquisa foi submetida à aprovação do Comitê de Ética em Pesquisa da Universidade Estadual de Campinas, conforme Parecer $\mathrm{n}^{\mathrm{o}} 066$ de 27 de março de 2007 e CAAE 0142.0.000.146-07 e segue os princípios estabelecidos pela resolução 196/96 do Ministério da Saúde.

Constitui-se em uma pesquisa avaliativa, de caráter formativo, com equipe de composição mista. Houve a participação de avaliadores internos, ligados ao programa de saúde do trabalhador e à vigilância do município, e avaliadores externos à universidade. Em reuniões preliminares e orientadoras da pesquisa, os avaliadores e os profissionais da Secretaria Municipal de Saúde de Campinas definiram, em conjunto, como seria a abordagem da questão e os critérios de avaliação que seriam utilizados pelos pesquisadores. Os critérios de avaliação, assim definidos pela equipe de avaliadores, consideraram quais seriam as ações de Visat desejáveis a serem desenvolvidas pelas equipes das UBS e este conjunto de ações seria o padrão ouro da avaliação. Procedeu-se à avaliação através da comparação desse conjunto de ações consideradas adequadas pelos avaliadores e o que se verificaria na realidade das UBS.

Assim, as ações esperadas de vigilância, que se constituíram em critérios de avaliação, seriam:

- Atendimentos dos trabalhadores acidentados e doentes;

- Registro das informações que pudessem indicar gravidade e frequência dos acidentes, como o tipo do acidente e a empresa, para investigação;

- Identificação de situações que necessitassem intervenção no local de trabalho;

- Intervenção no ambiente de trabalho;

- Avaliação dos resultados obtidos com a intervenção.

A partir desses critérios mais gerais, foram construídos indicadores de avaliação que ajudaram a definir o quanto as ações implementadas aproximaram-se ou afastaram-se das diretrizes da descentralização (WORTHEN; SANDERS; FITZPATRICK, 2004; SILVA, 2005).

O intuito foi avaliar o quanto as UBS reconhecem os acidentes e as doenças do trabalho como problema relevante que merece ser registrado em seus instrumentos de vigilância e como agem so- bre eles. Utilizamos como indicadores o número de acidentes e doenças do trabalho registrado na unidade de saúde; os percentuais de registros que permitiam identificar gravidade e frequência dos acidentes e, também, os percentuais desses trabalhadores segundo o seu vínculo formal ou informal com o mercado de trabalho. Escolhemos categorizar o atendimento do trabalhador segundo o vínculo com o mercado de trabalho em função do crescimento do número de trabalhadores informais na economia brasileira. Procuramos também verificar se as unidades desencadearam ou realizaram ações de Visat no local de trabalho a partir dos problemas de saúde relacionados ao trabalho, bem como se essas intervenções provocaram mudanças no processo de trabalho e no meio ambiente.

A equipe de avaliadores constituída para a pesquisa envolveu gestores e trabalhadores da Covisa, responsável pela coordenação do Sistema de Vigilância em Saúde no município; do Cerest, que coordena as ações de Saúde do Trabalhador em Campinas; e das equipes distritais de Visa, responsáveis por todas as ações de Visa na área do distrito de saúde, inclusive apoio técnico às UBS.

A coleta de dados foi feita em 2007 nos 49 Centros de Saúde existentes à época, tomando os dados do ano de 2006, com a finalidade de verificar a efetivação da descentralização da Visat nesse município (WORTHEN; SANDERS; FITZPATRICK, 2004; SILVA, 2005).

Os resultados obtidos, seguindo as definições de critérios e padrões de julgamento anteriormente definidos, foram apresentados novamente para apreciação do grupo de avaliadores, desta vez ampliado para os diferentes grupos de interessados e implicados da Secretaria de Saúde e da universidade. O intuito neste ponto da avaliação era o de que a avaliação pudesse ser utilizada pela gestão para implementação de medidas e correção de rumos.

Foram analisados os documentos produzidos por gestores e fóruns colegiados de diferentes níveis de gestão da Secretaria de Saúde de Campinas, relatórios de seminários e oficinas de trabalho e de conferências municipais de saúde, no período de 1990 a 2006, relativos à Visa e à Visat. Identificamos nesses documentos a declaração explícita da diretriz da descentralização dessas áreas para as UBS, como estratégia de reorganização dos serviços e de aproximação das pessoas às quais eles se destinam, na perspectiva da integralidade da atenção à saúde. Tomamos como base a diretriz de descentralização expressa nos documentos e procuramos verificar a sua efetivação nas UBS.

Para obter esses indicadores, tomamos como base o ano de 2006 e utilizamos o instrumento de registro de dados no nível local do Sistema de Vigilância Epidemiológica (SVE-2) e os registros das CATs, uma 
vez que deveriam ser registrados no SVE-2 todos os agravos sujeitos à notificação compulsória além de outros que fossem eleitos pela unidade de saúde como relevantes. No caso do acidente de trabalho, havia a orientação da Secretaria Municipal de Saúde de utilizar o SVE-2 para o seu registro. Além disso, algumas unidades adotaram outro livro similar para registrarem apenas as CATs.

Verificamos se as unidades utilizavam o SVE2 de maneira a registrar os dados relevantes para que pudesse conhecer a demanda que utiliza seus serviços e identificar sinais de alerta na situação epidemiológica.

Não utilizamos o Sistema Nacional de Agravos sob Notificação (Sinan), uma vez que, no ano de 2006, esses agravos não faziam parte do rol de doenças e agravos para os quais o Sinan estava preparado para processar.

Na mesma visita à unidade de saúde para o levantamento dos dados relatados acima, realizamos entrevistas semiestuturadas com o profissional responsável pela Visat no Centro de Saúde. As entrevistas tiveram como objetivo conhecer o modo como o Centro de Saúde desencadeava as ações de vigilância a partir do conhecimento dos casos de problemas de saúde relacionados ao trabalho.

Perguntamos se preenchiam o documento previdenciário denominado Comunicação de Acidentes de Trabalho e o instrumento epidemiológico, considerado o impresso oficial - o livro de registro de agravos notificáveis - SVE-2 ou livro similar, referentes ao acidente de trabalho. Perguntamos também se eram realizadas intervenções no local de trabalho, se essas possíveis intervenções, segundo os entrevistados, foram capazes de provocar mudanças no processo de trabalho desses trabalhadores expostos a algum risco e, ainda, se essas intervenções foram capazes de provocar mudanças no ambiente externo. Fizemos o registro sumário dos casos relatados em que houve intervenção.

Esse trabalho não se propôs a analisar a opinião de trabalhadores, atores externos ao SUS, particularmente devido à dificuldade operacional para coletar informações sobre trabalhadores nas áreas de abrangência das UBS, uma vez que não há representação específica de trabalhadores externos à rede de serviços de saúde nos conselhos locais de saúde.

\section{Resultados}

No período de estudo, o ano de 2006, foram registrados os dados das 49 UBS existentes no município de Campinas naquele momento. Verificamos que, dentre as unidades de saúde, apenas uma não preencheu a CAT, o que foi justificado pela falta do profissional médico naquela unidade, fazendo com que os trabalhadores fossem encaminhados ao Cerest para essa providência. A significativa maioria das unidades informou que preenchia as CATs.

Das 49 UBS, 43 registraram agravos relacionados ao trabalho no SVE-2 ou similar. Seis unidades não faziam registro em SVE-2 ou similar; estas unidades de saúde faziam apenas um registro administrativo das CATs, ou seja, um registro, com caráter burocrático, do envio desse documento à equipe de Visa que faria os encaminhamentos seguintes ao Cerest e ao INSS.

Das 43 unidades que faziam algum registro em SVE-2 ou similar, $25(58,14 \%)$ delas não registravam os dados clínicos do acidente, contra $18(41,86 \%)$ que o faziam. Em apenas 7 unidades $(14,28 \%)$ havia a informação quanto ao local de trabalho ou empresa empregadora desses trabalhadores que sofreram acidentes e em 36 unidades de saúde $(83,72 \%)$ não havia o registro da informação relativa ao local de trabalho.

Foram registrados nas UBS de Campinas, em 2006, um total de 12.853 agravos à saúde, sendo aqueles de notificação compulsória no Estado de São Paulo e no município de Campinas e também outros agravos que as unidades de saúde elegeram como relevantes. Deste total, foram registrados 1.420 eventos relacionados à saúde do trabalhador, representando $11,05 \%$ do total dos registros no período investigado.

A média (desvio padrão) dos registros de agravos à saúde do trabalhador foi de $28,98(28,55)$ casos por UBS no ano, a mediana foi de 22 . Os acidentes do trabalho representaram 96\% dessas notificações e as doenças relacionadas ao trabalho, apenas $4 \%$. O vínculo de trabalho era formal para 1.385 casos e apenas 3 eram informais. Não foi possível obter informação sobre o tipo de vínculo com o mercado de trabalho para 32 registros (Tabela $\mathbf{1}$ ).

Em 10 unidades básicas de saúde $(20,4 \%)$, houve relato de visitas e/ou intervenção nos locais de trabalho. Já em 39 unidades $(79,6 \%)$ não houve quaisquer relatos de visitas ou intervenções. De acordo com a opinião dos entrevistados, das intervenções relatadas, 4 provocaram mudanças no processo de trabalho, contra uma que não produziu mudança e cinco com informação ignorada. A repercussão das mesmas 10 intervenções sobre o ambiente externo foi relatada para 4. Duas intervenções foram consideradas improdutivas e não foi possível obter informação sobre as 4 restantes (Tabela 2 ). 
Tabela 1 Agravos de saúde e seus vínculos com o trabalho atendidos nas Unidades Básicas de Saúde de Campinas, SP, 2006

\begin{tabular}{lccccc}
\hline & \multicolumn{5}{c}{ Trabalhoformal } \\
\multicolumn{1}{c}{ Problema saúde } & Sim & Não & Ignorado & Total & $\%$ \\
\hline Acidente & 1.328 & 3 & 32 & 1.363 & 96 \\
Doença & 57 & 0 & 0 & 57 & 4 \\
Total & 1.385 & 3 & 32 & 1.420 & 100 \\
\hline
\end{tabular}

Fonte: Livro de registros epidemiológicos (SVE-2) das UBS

Tabela 2 Intervenções e influência de ações feitas por Unidades Básicas de Saúde que notificaram acidentes do trabalho em Campinas, SP, 2006

\begin{tabular}{lcc}
\hline \multicolumn{1}{c}{ Ações } & $n$ & $\%$ \\
\hline Realizou intervenção & 10 & 20,4 \\
sim & 39 & 79,6 \\
não & & \\
Mudou processo de trabalho & 4 & 8,2 \\
$\quad$ sim & 40 & 81,6 \\
não & 5 & 10,2 \\
ignorado & & \\
Mudou ambiente externo & & 8,2 \\
sim & 4 & 83,7 \\
não & 41 & 8,2 \\
ignorado & 4 & 100,0 \\
Total & 49 & \\
\hline
\end{tabular}

Fonte: entrevistas nas UBS

\section{Discussão}

Em razão da natureza e da complexidade do objeto a ser estudado, pareceu-nos adequada a utilização da triangulação de métodos proposta por Minayo, que possibilita uma abordagem múltipla e abrangente pela combinação de diferentes elementos: "a presença do avaliador externo", compondo com indivíduos que trabalham no programa uma equipe mista de avaliação, "as abordagens quantitativas e qualitativas e, de forma relevante, a análise do contexto, da história, das relações, das representações e a participação", permitindo melhor compreensão das diversas faces da realidade (MINAYO, 2006, p. 28).

Assim, utilizamos a triangulação de várias abordagens para apreensão da realidade: a combinação de elementos quantitativos para a construção de indica- dores, qualitativos e, ainda, o envolvimento de diferentes grupos de implicados, como gestores e trabalhadores da Covisa, do Cerest, das equipes distritais de Visa e das unidades de saúde, permitindo captar diversas visões e aspectos da descentralização da Visat para as UBS no município. Como é tarefa do processo avaliativo, os presentes resultados iluminam a questão estudada e oferecem ao gestor material relevante para o aprofundamento de ações e a correção de rumos que seja necessária.

Neste sentido, verificamos na pesquisa avaliativa empreendida que o atendimento a trabalhadores que sofreram acidente de trabalho vem sendo realizado por meio de cuidados médicos e de enfermagem nas UBS, incluindo o preenchimento da CAT e seu encaminhamento à Visa, ao Cerest e ao INSS, em consonância com as orientações da Secretaria Municipal de Saúde (CAMPINAS, 2006a). 
A partir da visita às UBS e do contato com os responsáveis pela atenção aos trabalhadores, pudemos notar que os funcionários das unidades referiram o cuidado em cumprir a burocracia no preenchimento desse documento, uma vez que ele representa implicações legais e previdenciárias para o trabalhador do mercado formal, conforme apontavam documentos da Secretaria Municipal de Saúde (CAMPINAS, 2006a; b; c) e os autores Cordeiro et al. (2005) e Wünsch-Filho (1999).

Constatamos, em resumo, que as unidades não faziam o registro do acidente ou da doença apresentados pelo trabalhador de maneira a registrar dados de interesse epidemiológico. A constatação da baixa qualidade da informação epidemiológica acerca dos acidentes de trabalho nas UBS é coerente com a afirmação de Medeiros (2001) de que houve uma piora na qualidade da informação a respeito dos acidentes de trabalho após a descentralização para as UBS.

A baixa média de atendimentos ao longo do ano de 2006, ou seja, menos de um atendimento por semana, sugere que não há uma sobrecarga de atendimentos dessa natureza nas UBS.

Podemos dizer que, de acordo com esses dados obtidos na pesquisa, os acidentes de trabalho (96\% das notificações) têm sido mais identificados por parte das unidades do que as doenças do trabalho (apenas $4 \%$ do total das notificações). As pessoas com doenças poderiam ser atendidas, sem, contudo, terem seu diagnóstico relacionado ao trabalho, o que levaria à subnotificação dessas doenças. Da mesma forma, em um estudo sobre os acidentes de trabalho no Brasil, utilizando os dados do INSS a partir das CATs - dados, portanto, referentes a trabalhadores do mercado formal - no período de 1970 a 1995, Wünsch-Filho (1999) verificou que as doenças profissionais respondiam por menos de $1 \%$ a cada ano da série histórica e que os acidentes de trabalho significam praticamente a totalidade dos registros.

Observamos que apenas o trabalhador do mercado formal teve o registro do seu acidente ou doença do trabalho feito sistematicamente na UBS. O trabalhador do mercado informal, como grupo social, estava à margem desses serviços de saúde no que se refere à identificação e ao registro do agravo à sua saúde como relacionado ao trabalho enquanto forma de indicar necessidades e possibilidades de intervenção sobre os riscos a que esses trabalhadores estão expostos nos ambientes de trabalho. Esses trabalhadores foram atendidos com queixas agudas, mas não tiveram o seu problema registrado e conduzido como algo relacionado ao trabalho e que necessitasse de uma intervenção do setor saúde. Conforme aponta Cecílio (2001), a superação da não integralidade a grupos específicos só poderá acontecer se ela for captada na sua singula- ridade, ou seja, no espaço individual da prestação do cuidado, no encontro do usuário - esse trabalhador do mercado informal - com o profissional da unidade de saúde e para que este possa, então, reconhecê-lo como portador de necessidades de saúde, também singulares. No mercado informal, devem estar presentes riscos e incidências ainda maiores do que no mercado formal, portanto, a Visat deve voltar seu foco para esse segmento dos trabalhadores (WÜNSCH-FILHO, 1999). Acreditamos que a "preferência" do registro dos eventos ocorridos com o trabalhador do mercado formal se deva à exigência burocrática da abertura da CAT para eles e também à desvalorização do registro epidemiológico do acidente de trabalho.

As intervenções nos ambientes de trabalho ocorridas a partir da identificação da ocorrência de acidentes de trabalho disseram respeito a orientações para mudanças de funções no trabalho e realização de ginástica postural, realização de exames periódicos, organização e limpeza do ambiente de trabalho e interdição de equipamento.

Podemos perceber que houve dificuldade para que a equipe da atenção primária desencadeasse ações de vigilância da saúde do trabalhador a partir da clínica que realizam. Ou seja, os trabalhadores são atendidos e a abordagem clínica não se transforma em ações de vigilância, limitando a compreensão do problema e as possibilidades de oferta de ações de saúde mais resolutivas.

Medeiros (2001) relata que, com a descentralização do atendimento de acidentes de trabalho para as UBS, houve piora na qualidade das intervenções coletivas. Os dados obtidos por essa pesquisa não permitem analisar essa afirmação, especialmente pela falta de possibilidade de comparação com o período anterior. Não é possível afirmar que todas as intervenções deveriam necessariamente provocar mudanças no ambiente externo, pois não há informação suficiente para essa análise. Podemos estar diante de uma limitação na abordagem dos ambientes de trabalho, pois, de acordo com os apontamentos de Minayo-Gomez e Lacaz (2005), o modelo tradicionalmente desenvolvido de atenção à saúde dos trabalhadores não incorpora componentes da questão ambiental, altamente relevantes na atualidade para a sociedade e que ainda carece de protagonismos dos trabalhadores tanto nos locais de trabalho, quanto nos espaços de exercício de cidadania.

Esse quadro nos permitiu dizer que os Centros de Saúde não realizavam e também não desencadeavam ou solicitavam a intervenção de outros níveis - Visa ou Cerest - baseados em dados epidemiológicos, seja porque não os registravam, não os buscavam ou não os tinham disponíveis. Quando ocorreu alguma intervenção, ela foi baseada na percepção dos problemas pelos 
profissionais da unidade, seja pela vulnerabilidade social em alguns poucos casos ou, ainda, por demanda do Cerest ou da Visa. Esta situação nos levou à necessidade de discutir a utilidade dos sistemas formais de informação para desencadear as ações necessárias à diminuição ou à eliminação de fatores nocivos à saúde. Sob esse ponto de vista, talvez fosse mais eficaz a presença de especialistas do Cerest e da Visa em visita periódica e diálogo mais frequente com as UBS sobre esse campo de atuação.

Os registros tradicionais da vigilância epidemiológica, como o SVE-2, devem se prestar a favorecer a identificação de situações de alerta. A definição de temas prioritários a serem trabalhados e de ações a serem desencadeadas poderia ser feita em espaços coletivos, considerando os diferentes conhecimentos trazidos para o grupo, entre eles, a informação epidemiológica gerada no serviço de saúde e o conhecimento específico do Cerest e da Visa (CAMPOS, 2003).

Nossos dados permitiram identificar que os profissionais reconhecem as ações de Visat como de responsabilidade das UBS, o que reforça o acerto do esforço de descentralização que vem sendo implementado. Identificamos, porém, necessidades de aprimoramento dessas ações, com o estabelecimento de fluxos, rotinas e procedimentos, capacitação e envolvimento das equipes, aproximação das equipes de Visa, do Cerest, da Covisa e dos Centros de Saúde, organização e disseminação da informação e reorganização das ações de intervenção.

As diretrizes da Secretaria Municipal de Saúde apontaram na direção da descentralização da Visa, englobando a Visat para as UBS, e não colocaram limites para isso. Portanto, era possível que as unidades realizassem essas ações plenamente, usando de criatividade e autonomia para interpretar localmente a diretriz da descentralização. Assim sendo, podiam propor ou realizar mecanismos e processos de trabalho locais para atender melhor às necessidades da população de sua área de abrangência, buscando a integralidade da ação, articulando ações de promoção, prevenção e a clínica ampliada, conforme apontam Merhy (1997) e Campos (2003).

Os Centros de Saúde poderiam ser considerados como "os olhos" do sistema de Vigilância da Saúde do Trabalhador na medida em que, conhecedores da sua realidade local, do seu território, conseguem identificar problemas e desencadear ações diretamente pelas suas equipes ou acionar outros níveis do sistema - as Visas ou Cerests - para o desenvolvimento dessas ações. Podemos dizer que a primeira etapa das ações de Visa - o conhecimento de um problema - pode ser cumprida pelo Centro de Saúde.
Na sequência, as outras ações, como a notificação, o registro, as medidas de intervenção e controle, a avaliação e a divulgação das informações, poderão ser realizadas direta e integralmente pela unidade básica se assim for definido e pactuado e se for provida a estrutura necessária para viabilizá-las. A saúde do trabalhador deve estar presente em todos os níveis do SUS, de forma integral e hierarquizada.

Contudo, não foi essa realidade que verificamos. O que observamos foi uma atuação parcial das unidades. Quando se analisa a forma como foi implantada a descentralização do atendimento dos acidentes de trabalho para as UBS, verificamos uma diferença significativa do que se pretendeu com a descentralização da vigilância: houve produção de manuais, estabelecimento de fluxos e competências, treinamentos e, sobretudo, acompanhamento por parte da equipe do Cerest de todas as equipes de Visa e UBS. Embora com problemas na sua execução, não há dúvida quanto à responsabilidade do Centro de Saúde em atender e dar os encaminhamentos para esses trabalhadores. No caso de desencadear outras ações de vigilância para além da notificação, isso já não ficou claro.

Concluímos que a declaração de uma diretriz não foi suficiente para que ela fosse implantada. É necessário que a declaração seja seguida de medidas para sua implantação e que esta tenha acompanhamento (MERHY, 1997), o que parece não ter acontecido. Entendemos que a capacitação e os acordos para se estabelecer as responsabilidades, os papéis e os fluxos, na linha da educação permanente, podem ser um meio eficaz de qualificação dessas equipes para assumirem a vigilância da saúde do trabalhador.

Vemos, também, uma cisão entre assistência e vigilância. Não seria o atendimento ao trabalhador uma primeira etapa do processo de vigilância sobre a sua saúde? Por que, então, essa primeira etapa não progride para as seguintes, que dariam conta de provocar mudanças naquela realidade dos ambientes de trabalho?

Vários autores (MINAYO-GOMEZ; THEDIM-COSTA, 1997; TEIXEIRA; PAIM; VILASBOAS, 2002; MINAYO-GOMEZ; LACAZ, 2005) indicam essa direção: Assistência e Vigilância deveriam andar juntas! À época da descentralização do atendimento dos acidentes de trabalho para as UBS, já era apontada como necessária a descentralização da intervenção em ambientes de trabalho, segundo Medeiros (2001). A dissociação das práticas de saúde pública e da assistência presente nos serviços de saúde não atende ao princípio da integralidade na atenção ampliada às necessidades de saúde dos trabalhadores (MATTOS, 2001). 
O que prevaleceu foi a oferta de ações com práticas fragmentadas, cristalizadas e organizadas de acordo com as rotinas tradicionais de cada serviço, sem buscar a construção de redes efetivas de serviços que possam dar, em conjunto, respostas mais adequadas às necessidades de saúde (CECÍLIO, 2001).

Essa fragmentação artificial que verificamos entre assistência e vigilância pode ter sido reforçada pela cristalização de concepções existentes, presentes à época da instituição dos centros de referência de saúde do trabalhador no país, que estabelecia para esses centros a atribuição de executar as ações da vigilância da saúde do trabalhador e, desta forma, podem ter desobrigado os Centros de Saúde da atuação de saúde coletiva e contribuído para o distanciamento dos Cerest em relação à rede básica de saúde (MINAYO-GOMEZ; LACAZ, 2005).

A integração das diferentes áreas da Vigilância e da Assistência só se dará através dos serviços que se propõem a atuarem efetivamente, pois o que de fato promove a integração não são as estruturas organizacionais, mas os problemas reais. Os exemplos das contaminações ambientais em postos de combustíveis, empresas químicas, depósitos de resíduos, nos quais trabalhadores, vizinhos e frequentadores são submetidos a diferentes rotas de exposição a produtos químicos, seja pelo contato direto com essas substâncias ou ainda pelo solo, pelo ar ou pela água, mostram-nos a necessidade da construção de processos de intervenção articulados entre as áreas de assistência à saúde e de vigilância epidemiológica, sanitária e ambiental destinadas a trabalhadores e outros grupos da população envolvida.

O grau da descentralização que se pode atingir deve ser pactuado respeitando-se as condições locais, sem perder a imagem da atuação ideal de uma UBS, que deveria realizar tudo o que fosse possível na sua área de abrangência. Desta forma, a busca da integralidade ampliada das ações em rede é um ideal que precisa ser construído, conforme nos falam Cecílio (2001) e Mattos (2001).

Todo o processo de descentralização das ações de saúde necessita de componentes estruturais, de gestão dos processos de trabalho e de avaliação dos resultados obtidos. Ela tem significado uma ferramenta potente para a organização da atenção integral, na medida em que pode, na capilarização das ações, perceber as necessidades de saúde, principalmente dos grupos mais vulneráveis.

O município de Campinas, no seu esforço de mais de três décadas de construção do Sistema de Saúde, desenvolveu-se ainda mais com a implantação do SUS e persegue a consolidação dos seus princípios norteadores. Porém, nem sempre as políticas sociais conseguem ser implantadas de forma linear. Sempre há contingências.

\section{Considerações finais}

O processo de avaliação formativa e participativa empreendido nas 49 UBS do município de Campinas, nas unidades de Visa e nos Cerests no ano de 2006, ainda que sem um componente fundamental, que seria a participação dos trabalhadores na avaliação, mostrou-se com muitas possibilidades de descortinar a complexidade das ações de saúde como no caso da Saúde do Trabalhador.

No sistema de saúde de Campinas, o processo de descentralização da Visat ocorreu de forma parcial para as UBS, pois as questões clínicas, via de regra, não se transformaram em ações de vigilância.

Observamos evidências de aspectos de descentralização efetiva de algumas ações, como o atendimento dos trabalhadores nos Centros de Saúde, principalmente quando se acidentam, indicando o acerto da diretriz da descentralização do atendimento dos acidentes de trabalho que não necessitam de atendimento hospitalar para a rede de atenção primária pela facilidade de acesso. Porém, quando se tratou de doenças relacionadas ao trabalho, o atendimento ocorreu em número menor e/ou não se buscou a relação causal com o trabalho. Este fato pode levar à subnotificação das doenças e, ainda, apontar para a dificuldade de se incluir, no cotidiano dos profissionais, o trabalho como um dos determinantes da doença. A preocupação com o atendimento dos trabalhadores acidentados ou doentes foi maior em relação aos cuidados oferecidos do que em relação a seu registro. $\mathrm{O}$ registro dos agravos foi realizado de maneira burocrática, sem privilegiar o caráter epidemiológico que favorecesse as ações de intervenção. Estas, quando aconteceram, foram desencadeadas pela sensibilidade dos profissionais de saúde ao problema e não motivadas pelo registro. A análise da situação de saúde por uma unidade básica poderia ser iniciada a partir dos dados gerados por ela própria e ser motivadora de ações de intervenção nos problemas identificados, o que definitivamente a aproximaria das necessidades advindas do território.

É necessário o aprofundamento de questões relativas ao sistema de informação, aos fluxos entre os diferentes componentes do sistema de saúde, à sua utilidade e ligação com os mecanismos de identificação e eleição de prioridades para atuação e intervenção nos locais de trabalho por parte da UBS, com o apoio dos serviços de vigilância e de saúde do 
trabalhador, e, principalmente, à clareza dos papéis dos diferentes níveis do Sistema Municipal na Visat.

A outra questão que merece aprofundamento diz respeito ao trabalhador sem vínculo formal com o mercado de trabalho. $\mathrm{O}$ atendimento clínico do trabalhador informal ocorreu nas unidades. Contudo, esse grupo não recebeu ação específica de vigilância para seu problema, o que contraria os princípios de universalidade, equidade e integralidade da atenção, que se concretizam na atenção às necessidades dos indivíduos. E, ainda que não haja formulações teóricas que abordem a questão dos trabalhadores do mercado informal, o problema segue batendo à porta das Unidades de Saúde e não pode ficar sem resposta. Há experiências, ainda que isoladas, de abordagem desses trabalhadores. Elas precisam ser incentivadas, desenvolvidas e discutidas amplamente. E, talvez, a partir da criatividade presente nos grupos que se dispõem a trabalhar com esse assunto complexo, surjam elementos que auxiliem no avanço para a solução desta questão relevante, que é a formulação de políticas específicas e o necessário acolhimento do trabalhador informal pelo sistema de saúde. Esse tema motivou a continuidade do estudo.

\section{Contribuição de autoria}

Balista, S. R. R.: realizou o trabalho de pesquisa como parte do programa de pós-graduação, incluindo a elaboração do projeto, o trabalho de campo, a análise dos dados, a redação da dissertação, do artigo e a revisão do original enviado para publicação. Santiago, S. M.: co-orientou o trabalho de pesquisa, em especial no que se refere às metodologias qualitativas. Contribuiu em todas as fases do projeto, especialmente na fase de elaboração e na análise das informações obtidas. A redação da dissertação e do artigo também contou com a colaboração da coautora. Corrêa Filho, H. R.: orientou o trabalho de pesquisa incluindo o delineamento do projeto, a submissão ao CEP-Unicamp e o planejamento da execução em campo. Compartilhou a busca de co-orientação qualitativa pela Professora Silvia Santiago, apoiou o desenvolvimento de contatos para o trabalho de campo, participou da análise dos dados primários, da redação da dissertação, do artigo e da revisão do original enviado para publicação.

\section{Referências}

ABRAHÃO, C. E. C. Municipalização, descentralização e vigilância à saúde em Campinas, 1988 - 1994. Disponível em: <http://www.carlosecabrahao.med.br/ saude/monografias/mono00004.htm>. Acesso em: 05 de out. 2007.

BRASIL. Ministério da Saúde. Portaria nº 2.437/GM, de 7 de dezembro de 2005. Dispõe sobre a ampliação e o fortalecimento da Rede Nacional de Atenção Integral à Saúde do Trabalhador - Renast no Sistema Único de Saúde - SUS. Disponível em: < http://bvsms.saude. gov.br/bvs/saudelegis/gm/2005/prt2437_07_12_2005. html>. Acesso em: 24 set. 2011.

CAMPINAS. Câmara Municipal. Lei nº 6.764 de 13 de novembro de 1991. Autoriza o Executivo a observar, no Município de Campinas, a legislação federal e estadual concernentes às ações de vigilância e fiscalização exercidas na promoção, proteção e recuperação da saúde e preservação do meio ambiente, e dá outras providências. Diário Oficial Municipal, 14 nov. 1991. p. 2-3. Campinas, 1991.

. Prefeitura Municipal de Campinas. Secretaria da Saúde. Programa de Saúde do Trabalhador. Síntese das ações em saúde do trabalhador desenvolvidas pela Secretaria Municipal de Saúde de Campinas. Campinas, 1997.

. Prefeitura Municipal de Campinas. Secretaria da Saúde. Centro de Referência de Saúde do Trabalhador. Manual de notificação de acidentes de trabalho e doenças ocupacionais através da CAT. Campinas, 2006a.

. Prefeitura Municipal de Campinas. Secretaria da Saúde. Centro de Referência de Saúde do Trabalhador. Plano de ação e metas em saúde do trabalhador. Campinas, 2006b.

. Prefeitura Municipal de Campinas. Secretaria da Saúde. Colegiado de Vigilância em Saúde. Subsídios para a $7^{\underline{a}}$ Conferência Municipal de Saúde: Saúde Coletiva, Vigilância em Saúde e Saúde Ambiental. Campinas, 2006c.

CAMPOS, G. W. S. Saúde paidéia, São Paulo: Hucitec, 2003.

CECÍLIO, L. C. O. As necessidades de saúde como conceito estruturante na luta pela integralidade e equidade na atenção. In: PINHEIRO, R.; MATTOS, R. A. (Org.). Os sentidos da integralidade na atenção e no cuidado à saúde. Rio de Janeiro: UERJ/IMS/Abrasco, 2001. p. 113-126. 
CORDEIRO, R. et al. O sistema de vigilância de acidentes do trabalho de Piracicaba, São Paulo, Brasil. Cadernos de Saúde Pública, Rio de Janeiro, v. 21, n. 5, p. 1574-1583, 2005.

MACHADO, J. M. H. Processo de vigilância em saúde do trabalhador. Cadernos de Saúde Pública, Rio de Janeiro, v. 13, n. 2, p. 33-45, 1997.

A propósito da vigilância em saúde do trabalhador. Ciência e Saúde Coletiva, Rio de Janeiro, v. 10, n. 4, p. 987-992, 2005.

MATTOS, R. A. Os sentidos da integralidade: algumas reflexões acerca de valores que merecem ser defendidos. In: PINHEIRO, R.; MATTOS, R. A. (Org.). Os sentidos da integralidade na atenção e no cuidado à saúde. Rio de Janeiro: UERJ/IMS/Abrasco, 2001. p. 39-64.

MEDEIROS, M. A. T. O Centro de Referência em Saúde do Trabalhador de Campinas: trajetória de uma experiência. 2001. 220 f. Dissertação (Mestrado)-Instituto de Filosofia e Ciência Humanas, Universidade Estadual de Campinas, Campinas, 2001.

MERHY, E. E. Em busca do tempo perdido: a micropolítica do trabalho vivo em saúde. In: ONOCKO, R.; MERHY, E. E. (Org.). Agir em saúde: um desafio para o público. São Paulo; Buenos Aires: Hucitec; Lugar Editorial, 1997. p. 71- 112.

MINAYO-GOMEZ, C.; LACAZ, F. A. C. Saúde do trabalhador: novas-velhas questões. Ciência e Saúde Coletiva, Rio de Janeiro, v. 10, n. 4, p. 797-807, 2005.

MINAYO-GOMEZ, C.; THEDIM-COSTA, S. M. F. A construção do campo da saúde do trabalhador: percurso e dilemas. Cadernos de Saúde Pública, Rio de Janeiro, v. 13, n. 2, p. 21-32, 1997.

MINAYO, M. C. S. Conceito de avaliação por triangulação de métodos. In: MINAYO, M. C. S.;
ASSIS, S. G.; SOUZA, E. R. (Org.). Avaliação por triangulação de métodos - abordagem de programas sociais. Rio de Janeiro: Fiocruz, 2006. p. 19-51.

SAGLIONI, I.; BALISTA, S. R. R. Harmonizar teorias e práticas: o fazer do município a experiência de Campinas. Campinas, 2004. (Trabalho não publicado).

SILVA-JUNIOR, A. G.; MERHY, E. E.; CARVALHO, L. C. Refletindo sobre o ato de cuidar da saúde. In: PINHEIRO, R.; MATTOS, R. A. (Org.). Construção da integralidade: cotidiano, saberes e práticas em saúde. Rio de Janeiro: IMS/UERJ/Abrasco, 2003. p. 113-128.

SILVA, L. M. V. Conceitos, abordagens e estratégias para avaliação em saúde. In: HARTZ, Z. M. A.; SILVA, L. M. V. (Org.). Avaliação em Saúde: dos modelos teóricos à prática na avaliação de programas e sistemas de saúde. Salvador: EDUFBA; Rio de Janeiro: Fiocruz, 2005. p. 15-39.

TEIXEIRA, C. F.; PAIM, J. S.; VILASBOAS, A. L. Promoção e vigilância da saúde. Salvador: Centro de Estudos e Projetos em Saúde/Instituto de Saúde Coletiva, 2002.

VILELA, M. F. G. Da "moça da vigilância” ao núcleo de saúde coletiva na unidade básica de saúde: o que há de novo no modelo assistencial de Campinas? 2005. 257f. Tese (Doutorado em Saúde Coletiva)-Faculdade de Ciências Médicas, Universidade Estadual de Campinas, Campinas, 2005.

WORTHEN, B. R.; SANDERS, J. R.; FITZPATRICK, J. L. Avaliação de programas. São Paulo: Gente, 2004.

WÜNSCH-FILHO, V. Reestruturação produtiva e acidentes de trabalho no Brasil: estrutura e tendências. Cadernos de Saúde Pública, Rio de Janeiro, v. 15, n. 1, p. 41-51, 1999. 